XX Congreso de Ecuaciones Diferenciales y Aplicaciones

X Congreso de Matemática Aplicada

Sevilla, 24-28 septiembre 2007

(pp. 1-8)

\title{
Phase portraits of separable Hamiltonian systems
}

\author{
A. Guillamon ${ }^{1}, \underline{\text { Ch. Pantazi }}^{2}$, \\ ${ }^{1}$ Dept. de Matemàtica Aplicada I, Universitat Politècnica de Catalunya, Dr. Maraón n.44-50, 08028, \\ Barcelona, Catalonia, Spain,E-mail: antoni.guillamon@upc.edu. \\ 2 Dept. de Matemàtica Aplicada I, Universitat Politècnica de Catalunya, Avda. Diagonal 647 08028 \\ Barcelona E-mails: chara.pantazi@upc.edu.
}

\section{Resumen}

We study some generalizations of potential Hamiltonian systems $\left(H(x, y)=y^{2}+\right.$ $F(x)$ ) with one degree of freedom. In particular, we are interested in Hamiltonian systems with Hamiltonian functions of type $H(x, y)=F(x)+G(y)$ arising in applied mechanical problems. We present an algorithm to plot the phase portrait (include the behavior at infinity) of any Hamiltonian system of type $H(x, y)=F(x)+G(y)$, where $F$ and $G$ are arbitrary polynomials. We are able to give the full description in the Poincaré disk according to the graphs of $F$ and $G$, extending the well-known method for the "finite" phase portrait of potential systems.

\section{Introduction}

The algorithm to plot the finite phase portrait of potential systems is a well-known and classical example of qualitative theory of ordinary differential equations. The clue to be able to topologically classify this type of Hamiltonian systems is, obviously, the simplicity of its energy function,

$$
H(x, y)=\frac{y^{2}}{2}+F(x)
$$

with $F \in C^{1}(\mathbb{R})$ (see Figure 1).

The fact that $H$ "depends" basically on $F(x)$ allows to relate the phase portrait of $\left\{x^{\prime}=-H_{y}(x, y), y^{\prime}=H_{x}(x, y)\right\}$ with the graph of the one-variable function $F(x)$. This kind of reduction was also explored in [2] for Hamiltonian systems of type $H(r, \theta)=$ $r^{2} / 2+r^{n+1} g(\theta)$.

The aim of this work is to extend this type of results to Hamiltonian systems depending of more than one function. We have chosen a natural generalization of potential systems, those with energy function $H(x, y)=F(x)+G(y)$, which covers a wide range 


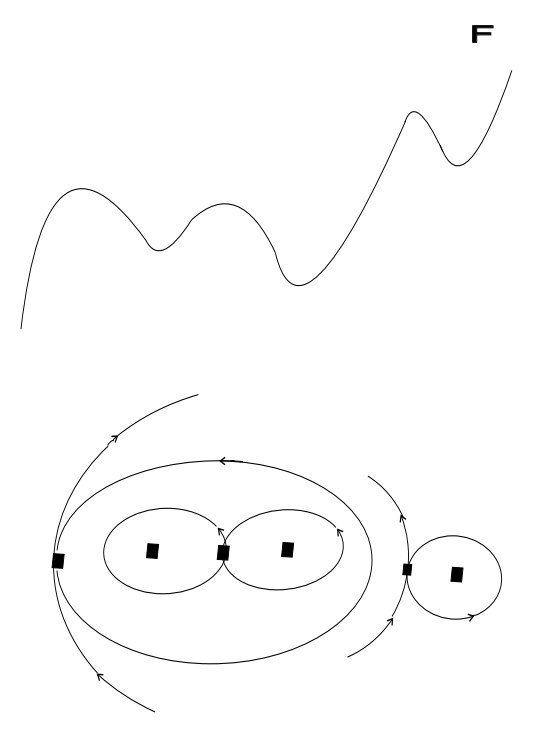

Figura 1: Finite representation of the phase portrait of a potential systems; minima of $F$ coincide with center points while maxima coincide with saddles.

of conservative physical models. We provide an algorithm to obtain the global (along the paper, "global" means that includes the behaviour at infinity) phase portrait of polynomial Hamiltonian systems of the form

$$
x^{\prime}=-G^{\prime}(y), \quad y^{\prime}=F^{\prime}(x),
$$

where $F$ and $G$ are arbitrary polynomials. We will refer to this vector field as $X_{H}$. Our description includes the classical classification of finite critical points of smooth potential systems.

To fix notation, we write:

$$
F^{\prime}(x)=a_{n} x^{n}+\cdots+a_{1} x, \quad a_{n} \neq 0 ; \quad G^{\prime}(y)=b_{m} y^{m}+\cdots+b_{1} y, \quad b_{m} \neq 0 .
$$

In the next two sections we provide the results that support the algorithm. For the sake of space, this document only presents the main stream to apply the algorithm, but does not cover all the proofs and details.

\section{Bounded dynamics}

Next two results are useful to determine the behaviour of bounded orbits of the system (1).

Proposition 1 (Finite critical points) Let $P=\left(x_{0}, y_{0}\right)$ be a finite singular point of system (1). Then,

(a) $P$ is a saddle if and only if $F$ has a maximum (resp., a minimum) at $x_{0}$ and $G$ has a minimum (resp., a maximum) at $y_{0}$. 
(b) $P$ is a center point if either $F$ has a maximum at $x_{0}$ and $G$ has a maximum at $y_{0}$ or $F$ has a minimum at $x_{0}$ and $G$ has a minimum at $y_{0}$.

(c1) $P$ is a cusp point if either $F$ has an inflection point at $x_{0}$ or $G$ has an inflection point at $y_{0}$.

(c2) If $F$ has an inflection point at $x_{0}$ and $G$ has an inflection point at $y_{0}$, then $P$ is a critical point formed by the union of two hyperbolic sectors.

Saddle points will be denoted by $S$; centers arising from two maxima will be denoted by $C^{-}$, while those coming from two minima will be denoted by $C^{+}$. All other types of finite critical points will be denoted by $D$.

The bounded separatrices of a saddle organize themselves according to the rules of the following proposition.

\section{Proposition 2 (Bounded separatrices)}

- Any center of type $C^{+}$is embraced by the proximal separatrices of the neighbouring saddle $S^{*}$ which energy level satisfies

$$
H\left(S^{*}\right)=\min \left\{S: H(S)>H\left(C^{+}\right)\right\} .
$$

- Any center of type $C^{-}$is embraced by the proximal separatrices of the neighbouring saddle $S^{*}$ which energy level satisfies

$$
H\left(S^{*}\right)=\operatorname{máx}\left\{S: H(S)<H\left(C^{+}\right)\right\} .
$$

- If the four separatrices of a saddle $S$ embrace the period annuli of a set of centers $\mathcal{C}:=\left\{C_{1}, \ldots, C_{n}\right\}$, then $\mathcal{C} \cup S \cup W^{u, s}(S)$ can be treated as a new center with the same energy level than $S$.

\section{Unbounded dynamics}

In order to study the behaviour of system (1) near infinity we use Poincaré compactification (see for instance [3]). If we call $\{X, Y, Z\}$ the coordinates in which the sphere is expressed, the equator lies on $Z=0$. Along the paper we are going to use both charts $\left(U_{1}, F_{1}\right)(\{X>0\})$ and $\left(U_{2}, F_{2}\right)(\{Y>0\})$ of the vector field (1) extended to the sphere. Additionally, we denote by $\left(V_{1}, G_{1}\right)(\{X<0\})$ and $\left(V_{2}, G_{2}\right)(\{Y<0\})$ the two charts on the opposite side of $\left(U_{1}, F_{1}\right)$ and $\left(U_{2}, F_{2}\right)$ respectively.

Depending on the sign and parity of $n-m$ we will use either chart, as it can be appreciated from the following cases:

\section{Case 1: $n>m$.}

In the $\left(U_{1}, F_{1}\right)$ chart the vector field takes the form

$$
\left\{\begin{aligned}
z_{1}^{\prime} & =z_{2}^{n}\left(z_{1} G^{\prime}\left(\frac{z_{1}}{z_{2}}\right)+F^{\prime}\left(\frac{1}{z_{2}}\right)\right) \\
& =a_{n}+a_{n-1} z_{2}+\cdots+a_{1} z_{2}^{n-1}+b_{m} z_{1}^{m+1} z_{2}^{n-m}+\cdots+b_{1} z_{1}^{2} z_{2}^{n-1} \\
z_{2}^{\prime} & =z_{2}^{n+1} G^{\prime}\left(\frac{z_{1}}{z_{2}}\right)=z_{2}^{n-m+1}\left(b_{m} z_{1}^{m}+b_{m-1} z_{1}^{m-1} z_{2}+\cdots+b_{1} z_{1} z_{2}^{m-1}\right) .
\end{aligned}\right.
$$


We note that there are no critical points on $\left\{z_{2}=0\right\}$ since $a_{n} \neq 0$.

In the $\left(U_{2}, F_{2}\right)$ chart the vector field can be written as

$$
\left\{\begin{aligned}
z_{1}^{\prime} & =-z_{2}^{n}\left(G^{\prime}\left(\frac{1}{z_{2}}\right)+z_{1} F^{\prime}\left(\frac{z_{1}}{z_{2}}\right)\right) \\
& =-b_{m} z_{2}^{n-m}-\cdots-b_{1} z_{2}^{n-1}-a_{n} z_{1}^{n+1}-\cdots-a_{1} z_{1}^{2} z_{2}^{n-1}, \\
z_{2}^{\prime} & =-z_{2}^{n+1} F^{\prime}\left(\frac{z_{1}}{z_{2}}\right)=-a_{n} z_{1}^{n} z_{2}-\cdots-a_{1} z_{1} z_{2}^{n},
\end{aligned}\right.
$$

which has a unique critical point, $z_{1}=z_{2}=0$, on $\left\{z_{2}=0\right\}$.

Case 2: $n=m$

On the $\left(U_{1}, F_{1}\right)$ chart, the vector field becomes

$$
\left\{\begin{array}{l}
z_{1}^{\prime}=\left(a_{n}+b_{n} z_{1}^{n+1}\right)+\left(a_{n-1}+b_{n-1} z_{1}^{n}\right) z_{2}+\cdots+\left(a_{1}+b_{1} z_{1}^{2}\right) z_{2}^{n-1} \\
z_{2}^{\prime}=z_{2}\left(b_{n} z_{1}^{n}+\cdots+b_{1} z_{1}\right) .
\end{array}\right.
$$

We note that critical points at infinity are given by $z_{1}^{n+1}=-\frac{a_{n}}{b_{n}}$.

On the $\left(U_{2}, F_{2}\right)$ chart the vector field is written

$$
\left\{\begin{array}{l}
z_{1}^{\prime}=-b_{n}-b_{n-1} z_{2}-\cdots-b_{1} z_{2}^{n-1}-a_{n} z_{1}^{n+1}-a_{n-1} z_{1}^{n} z_{2}-\cdots-a_{1} z_{1}^{2} z_{2}^{n-1} \\
z_{2}^{\prime}=-z_{2}\left(a_{n} z_{1}^{n}+\cdots+a_{1} z_{1} z_{2}^{n-1}\right)
\end{array}\right.
$$

and the critical points at infinity are given by $z_{1}^{n+1}=-\frac{b_{n}}{a_{n}}$.

When $n$ is odd and $\frac{b_{n}}{a_{n}}<0$, both (5) and (6) have 4 critical points at infinity, which coincide; if $\frac{b_{n}}{a_{n}}>0$, there are no critical points at infinity. When $n$ is even, both (5) and (6) have 2 critical points at infinity, which coincide.

Case 3: $n<m$. After an appropriate change of variables, this case is the equivalent to Case 1. Without loss of generality, then, we will omit its study and concentrate on system (6), defined on chart $\left(U_{2}, F_{2}\right)$.

One of the crucial steps to obtain the global phase portrait is to determine which separatrices tend (both for positive and negative times) to critical points at infinity. For this purpose, we need to distinguish special maxima and minima of functions $F$ and $G$, which apply for the case that $S=\left(x^{*}, y^{*}\right)$ is a saddle:

Definition 1 Given a critical point $S=\left(x^{*}, y^{*}\right)$ of system (1), we say that:

- $x^{*}$ satisfies the property $l$ (resp., $\left.\mathcal{L}\right)$ if $F\left(x^{*}\right)$ is a minimum (resp., maximum) of $F$ and $F\left(x^{*}\right)<F(x)$ (resp., $F\left(x^{*}\right)>F(x)$ ) for each $x<x^{*}$.

- $x^{*}$ satisfies the property $r$ (resp., $\mathcal{R}$ ) if $F\left(x^{*}\right)$ is a minimum (resp., maximum) of $F$ and $F\left(x^{*}\right)<F(x)$ (resp., $F\left(x^{*}\right)>F(x)$ ) for each $x>x^{*}$.

- $y^{*}$ satisfies the property d (resp., $\left.\mathcal{D}\right)$ if $G\left(y^{*}\right)$ is a minimum (resp., maximum) of $G$ and $G\left(y^{*}\right)<G(y)$ (resp., $G\left(y^{*}\right)>G(y)$ ) for each $y<y^{*}$.

- $y^{*}$ satisfies the property $u$ (resp., $\left.\mathcal{U}\right)$ if $G\left(y^{*}\right)$ is a minimum (resp., maximum) of $G$ and $G\left(y^{*}\right)<G(y)$ (resp., $G\left(y^{*}\right)>G(y)$ ) for each $y>y^{*}$.

Additionally, we define the following properties: 
- The saddle $S^{*}=\left(x^{*}, y^{*}\right)$ satisfies property (h1) if $x^{*}$ satisfies both the properties $r$ and $l$. Analogously, we will say that it satisfies property (H1) if $x^{*}$ satisfies both the properties $\mathcal{R}$ and $\mathcal{L}$.

- The saddle $S^{*}=\left(x^{*}, y^{*}\right)$ satisfies property (h2u) (resp., (H2U), (H2d) or (H2D)) if $y^{*}$ satisfies property $u$ (resp. $\mathcal{U}, d$ or $\mathcal{D}$ ) and its maximal (resp., maximal, minimal or minimal) with respect to this property.

Finally, we say that $(x(t), y(t))$ is an upper (resp., lower) separatrix of the saddle $S$ if there exists $\epsilon>0$ such that for $\left|y(t)-y^{*}\right|<\epsilon$, we have $y(t)>y^{*}$ (resp., $\left.y(t)<y^{*}\right)$.

Next result (and the corollaries not shown here) gives the behavior of the main separatrices that reach or come from a critical point at infinity.

Proposition 3 Let $S=\left(x^{*}, y^{*}\right)$ be a finite saddle point. Then:

- If $S$ satisfies the properties (h1) and (H2U), or (H1) and (H2u), the upper separatrices of $S$ form an elliptic sector $E$ at $+\infty$ (that is, the critical point of the vector field on chart $\left(U_{2}, F_{2}\right)$ on $\left.\left\{z_{2}=0\right\}\right)$.

- If $S$ satisfies the properties (h1) and (H2D), or (H1) and (H2d), the lower separatrices of $S$ form an elliptic sector $E$ at $-\infty$ (that is, the critical point of the vector field on chart $\left(V_{2}, G_{2}\right)$ on $\left.\left\{z_{2}=0\right\}\right)$, see Figure 4 .

Finally, for the infinite critical points we can use Hartmann Theorem in case they are hyperbolic. However, since the degrees $n, m$ and the coefficients $a_{n}$ and $b_{m}$ give all the information about the index of the vector field on $\mathbb{R}^{2}$, we can deduce the index of the infinite critical points from Poincaré-Hopf Theorem. Then, with the help of Proposition 3 and additional reasonings, we can give the topological classification of all infinite critical points.

More precisely the information about the finite index allows to establish the following result:

Proposition 4 Suppose first that $n-m$ is odd and let $q_{\infty}$ a critical point on the equator of the Poincaré sphere. Then,

- If $q_{\infty}$ does not have elliptic sectors, $q_{\infty}$ is a node.

- If $q_{\infty}$ has one elliptic sectors, $q_{\infty}$ is the union of this elliptic sector and a hyperbolic one, each of them lying on a different hemisphere of the Poincaré sphere (see Figure 2)(a).

Suppose now that $n-m$ is even. Then:

- If $i_{\mathbb{R}^{2}}=-1, q_{\infty}$ is the union of two elliptic sectors (see Figure 2)(b).

- If $i_{\mathbb{R}^{2}}=1, q_{\infty}$ is the union of two hyperbolic sectors (see Figure 2)(c). 


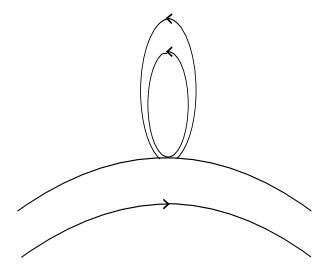

Elliptic and

hyperbolic sectors

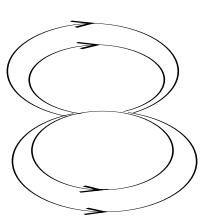

Two elliptic sectors

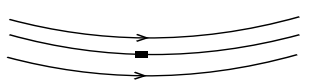

hyperbolic

sectors

Figura 2: Different types of critical points on the equator of the Poincaré sphere.

\section{Algorithm}

Combining propositions 1, 2, 3 and 4 one can describe the complete phase portrait on the Poincare sphere. In Figures 3 and 4 we give a couple of examples, explaining the whole procedure to build up the global phase portrait.

\section{Agradecimientos}

Partially supported by the DGES grant number MTM2005-06098-C02-1, the CONACIT grant number 2005SGR-986 and the MCyT/FEDER grant MTM2006-00478.

\section{Referencias}

[1] S. Diliberto, On systems of ordinary differential equations, Contributions to the Theory of Nonlinear Oscillators 1 (1950), 1-38.

[2] A. Gasull, A. Gulllamon, V. Mañosa Phase portrait of Hamiltonian systems with homogeneous nonlinearities, Nonlinear Analysis: Theory, Methods \& Applications 42 (2000), 679-707.

[3] J. Sotomayor, Liçoes de equaçoés diferenciais ordinárias, IMPA, Rio de Janerio, 1979. 

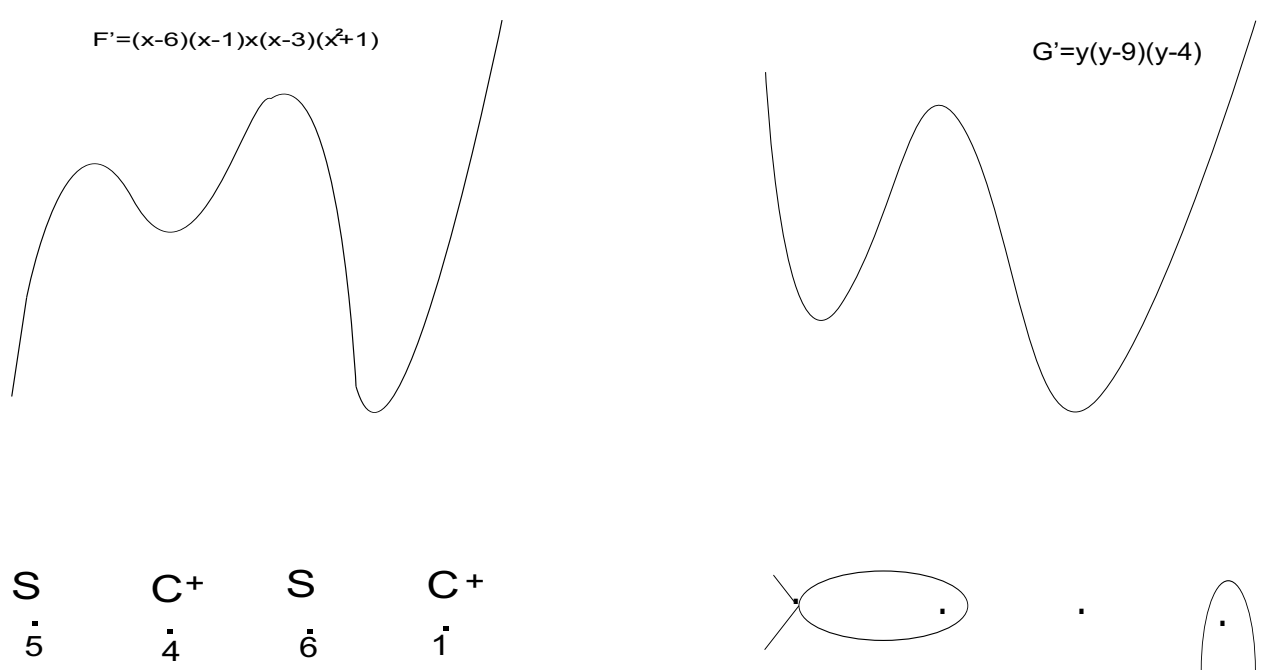

$\begin{array}{rrrr}11 & 10 & 12 & 3 \\ \mathrm{C}^{-} & \mathrm{S} & \mathrm{C}^{-} & \mathrm{S}\end{array}$

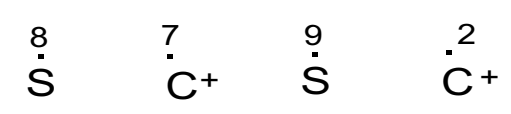
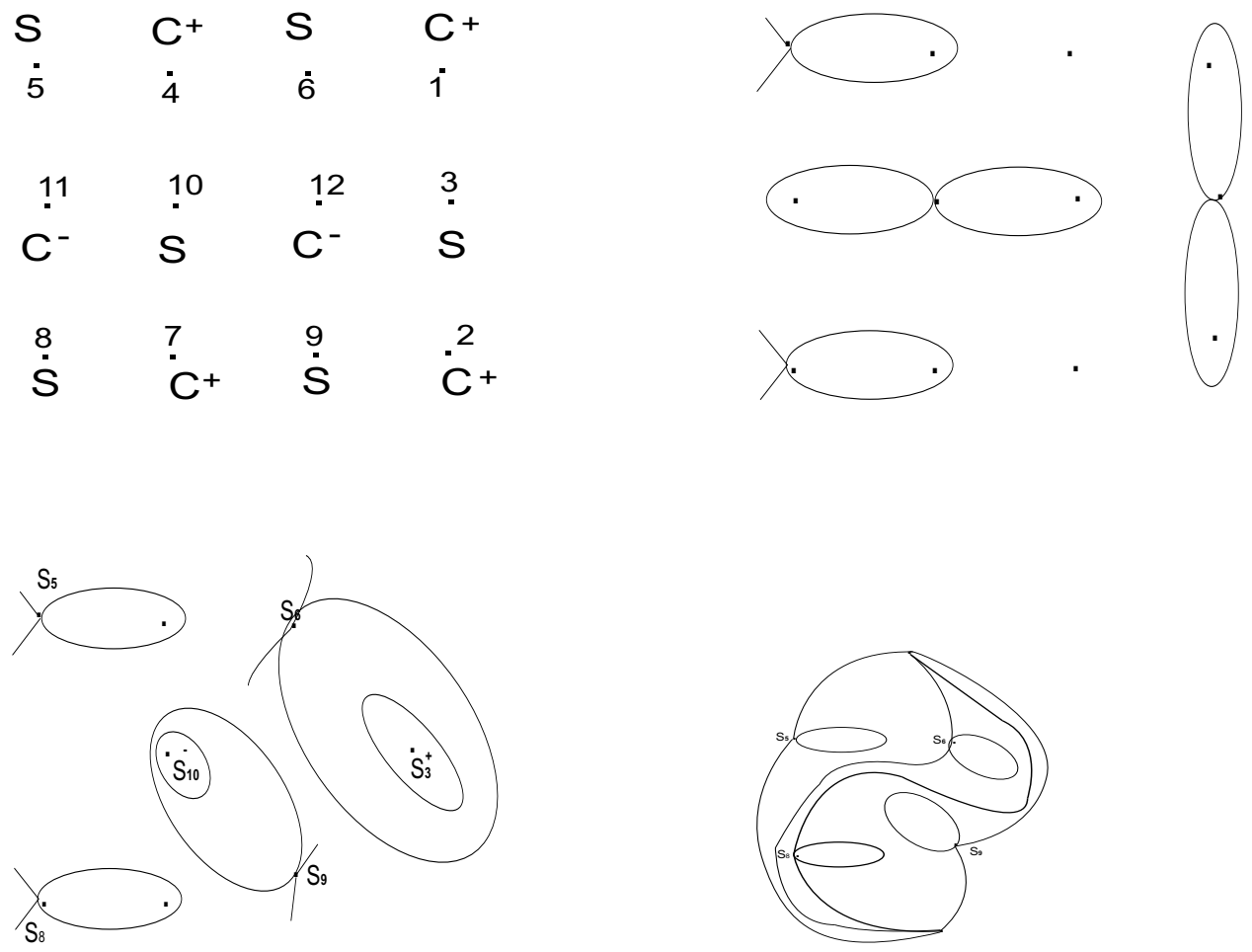

Figura 3: The saddles $S_{10}$ and $S_{3}$ become $S_{10}^{-}$and $S_{3}^{+}$respectively, see Proposition 2. There is a connection between each of the saddles $S_{9}, S_{8}, S_{6}, S_{5}$ and a critical point at infinity. Critical points at infinity are nodes, see Proposition 4. 


\section{$F^{\prime}=(x-4)(x-3)(x+5)$}
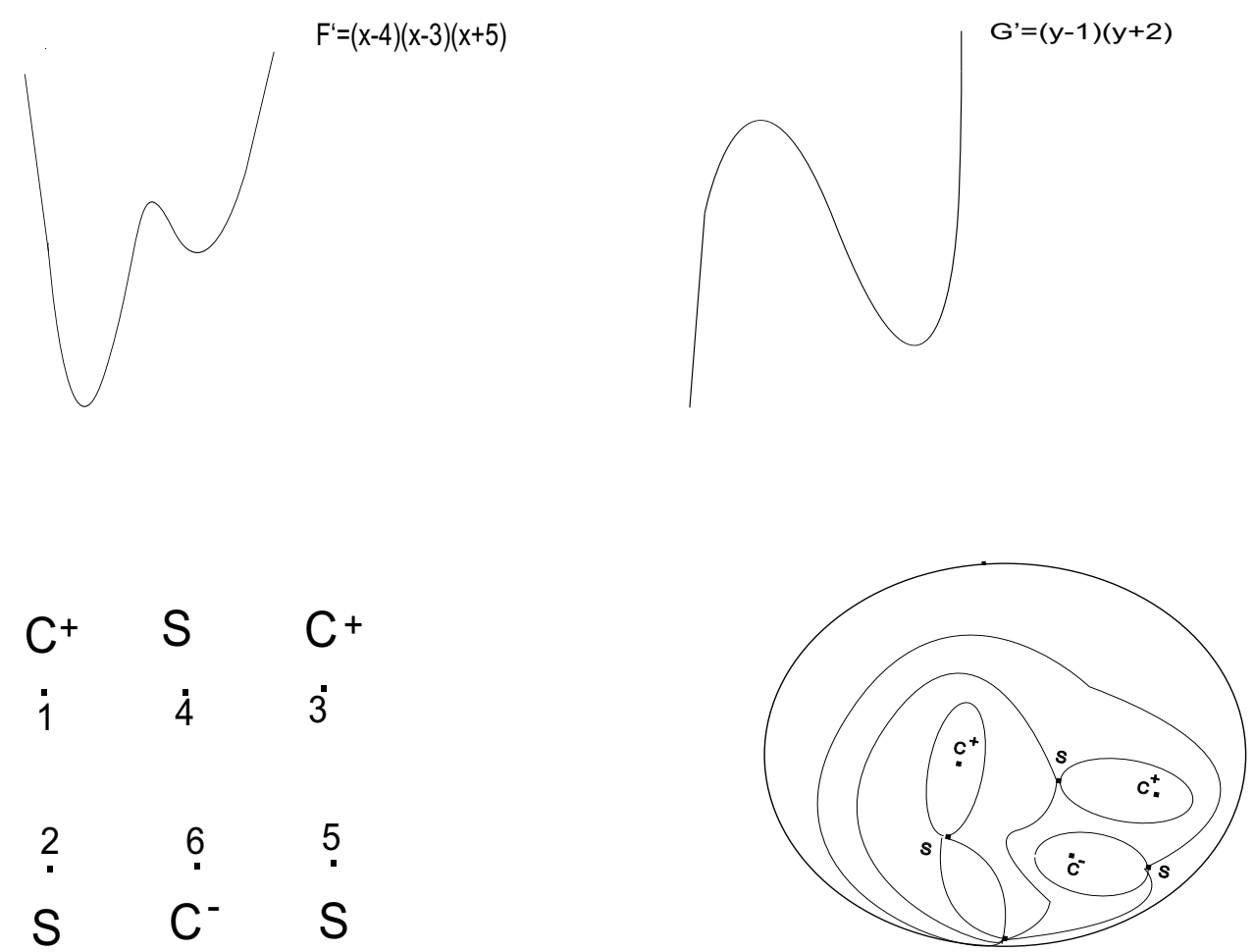

Figura 4: The saddle $S_{2}$ satisfies the properties (h1) and (H2D). From Proposition 3, the lower separatrices of the saddle $S_{2}$ form an elliptic sector at $-\infty$ and so $S_{2}$ becomes $S_{2}^{+}$. Then, the saddle $S_{4}$ embraces the "closed heteroclinic loop" $S_{2}^{+}$and so $S_{4}$ becomes $S_{4}^{+}$. At the end, the saddle $S_{5}$ embraces $S_{4}^{+}$. Additionally, the singular point at infinity is a union of elliptic and hyperbolic sectors, see also Figure 2(a). 